\title{
Posible influencia de los rametes en la estructura poblacional y distribución del árbol gigante Warszewiczia uxpanapensis, Cordillera de Tilarán, Costa Rica
}

\author{
Liz Brenes Cambronero ${ }^{1}$ \& José Francisco Di Stéfano ${ }^{2}$ \\ 1 Programa de Investigaciones del Bosque Premontano, Sede de Occidente, Universidad de Costa Rica, Apdo. Postal \\ 111-4250, San Ramón, Costa Rica, Fax 50644574 01; lmbrenes@cariari.ucr.ac.cr \\ 2 Escuela de Biología, Universidad de Costa Rica, 2060 San José, Costa Rica; jdistefa@cariari.ucr.ac.cr
}

Recibido 07-VII-2003. Corregido 13-XII-2005. Aceptado 10-V-2006.

\begin{abstract}
Possible influence of ramets on the population structure and distribution of the giant tree Warszewiczia uxpanapensis, Tilarán Mountain Range, Costa Rica. The distribution and population structure of Warszewiczia (=Elaeagia) uxpanapensis, a tree that reaches a height of $45 \mathrm{~m}$, a DBH of $1.40 \mathrm{~m}$ and $8 \mathrm{~m}$ tall buttresses, was studied on two 1-ha plots of premontane pluvial forest. It had a frequency of $49 \%$ and a mean dominance of $6.5 \%$. The adult distribution was random (mean distance to the nearest neighboring tree in plot 1, it was $14.26 \mathrm{~m}, \mathrm{n}=15$; Clark-Evans Index $(\mathrm{IC}-\mathrm{E})=1.07$; in plot 2, it was $15.33 \mathrm{~m}, \mathrm{n}=18, \mathrm{IC}-\mathrm{E}=1.34$,). The seedlings represented $60.3 \%$ of the sampled population (plot $1, n=94$; plot $2, n=85$ ). This species has a very high capability of propagating itself vegetatively through clones ("ramets"), which influence its distribution and population structure. With a ramet density in plot 1 of 0.8 and in plot 2 of $0.3 / 100 \mathrm{~m}^{2}$, its propagation could be from fallen trees or branches (which also create clearings in the forest) or from standing trees and even live branches that produce adventitious roots. Rev. Biol. Trop. 54 (4): 1179-1188. Epub 2006 Dec. 15.
\end{abstract}

Key words: clone, damage, population structure, ramets, Rubiaceae, vegetative propagation.

En condiciones naturales, muchas plantas pueden propagarse vegetativamente por tallos rastreros, estolones, rizomas o raíces (Barnes 1969, Sakai y Sharik 1988, Harada et al. 1997), a partir de yemas laterales de ramas (Gartner 1989, Brenes y Di Stéfano 1996-1997) o troncos caídos (Botina 1990, Brenes y Di Stéfano 1996-1997). Estos clones pueden representar una estrategia efectiva cuando las condiciones de reproducción por semillas y establecimiento de las plántulas (genete) son difíciles. En los trópicos esta condición se presenta en ecosistemas con algún tipo de estrés y en bosques secundarios (Richards 1996). Además de los competidores, las plantas clonales muestran plasticidad morfológica en su crecimiento, que es considerada importante para la eficiente obtención de recursos (Streitwolf-Engel et al. 1997).
Una vez establecidos, los brotes pueden formar unidades independientes o rametes (Alpert 1996). Esta propagación clonal tiene repercusiones en la estructura demográfica y genética poblacional en un sitio determinado, logrando desarrollar una colonia de plantas fisiológicamente independientes con el mismo genotipo (Begon y Mortimer 1992).

Por ejemplo, se estudió Tetramerista glabra (en Borneo, Indonesia) un árbol dominante del dosel en ecosistemas pantanosos, el cual produce un "banco de rametes" que funciona parecido a un banco de plántulas y así facilita la producción de reservorios de rametes, inclusive llega a dominar el reclutamiento en estadios juveniles de algunas especies (Gavin y Pearl 1999). En Canadá y Norte de Estados Unidos, Populus tremuloides y $P$. grandidentata forman clones a partir del sistema radicular (Barnes 1969). 
Richards (1996) señala a Dimorphandra conjugata, Cyrilla racemiflora y Grewia coriacea, los cuales también se propagan por rametes.

Elaeagia pastoensis D. Lorence (árbol encontrado en Colombia) produce rametes a partir de troncos o ramas caídas (Botina 1993) al igual que lo señalan Schöers et al. (1996) en E. auriculata (árbol en la Reserva Biológica Alberto Manuel Brenes -ReBAMB). Brenes y Di Stéfano (1996-1997) lograron que estacas de Warszewiczia uxpanapensis subsp. meridionalis (Sin. Elaeagia)(Lorence) (Taylor 2001) desarrollaran hojas y raíces en condiciones de invernadero.

El objetivo principal del presente estudio fue determinar la estructura y distribución espacial poblacional de $W$. uxpanapensis en un bosque pluvial premontano, y el posible aporte que puede hacer la propagación por rametes a estos aspectos. Además, se evaluaron los daños y la epifilia en las plántulas y brinzales.

\section{MATERIALES Y MÉTODOS}

Este trabajo se realizó en el período de enero 1997 a marzo 1998, en la Reserva Alberto Manuel Brenes, ubicada en la Cordillera de Tilarán,

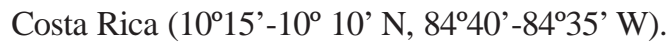
Dicho lugar presenta precipitación anual total de $3500 \mathrm{~mm}$ y temperatura anual promedio de 21 ${ }^{\circ} \mathrm{C}$ (Brenes 1999). La zona de vida corresponde al bosque pluvial premontano de acuerdo con el mapa de Bolaños y Watson (1993).

Para el estudio de la estructura poblacional y la distribución espacial, se establecieron dos parcelas cuadradas de 1 ha cada una, divididas en subparcelas de $10 \times 10 \mathrm{~m}$, donde se aseguró la presencia de individuos de $W$. uxpanapensis (Reg. No.58474 USJ, Herbario de la Escuela de Biología, Universidad de Costa Rica). Éstas se encontraban cercanas a la Estación Biológica en la cuenca del río San Lorencito, a una altitud de unos 850 msnm, separado en línea recta por espacio aprox. de $1 \mathrm{~km}$. Ambas presentaban fuertes pendientes.

Se registraron árboles, juveniles y plántulas, bajo las siguientes categorías diamétricas y de altura: $\geq 10 \mathrm{~cm}$ de DAP (adultos), $<10 \mathrm{~cm}$ a $5 \mathrm{~cm}$ DAP (juveniles), $<5 \mathrm{~cm}$ de DAP y $>1.5 \mathrm{~m}$ de altura (latizales), $\mathrm{y} \leq 1.5 \mathrm{~m}$ altura (que agrupó plántulas y brinzales); esta última se subdividió en cinco subclases basales: 1 a 10 , de 11 a 20, 21 a 30,31 a 40 y $>41 \mathrm{~mm}$. A cada individuo se le midió la altura (estimada con un clinómetro) DAP (diámetro a $1.35 \mathrm{~m}$ del suelo o arriba de las gambas), altura de gambas, el largo y ancho de la copa. A los latizales, brinzales y plántulas se les midió la altura (o longitud) y diámetro de la base del tallo (DAB). También se registraron los árboles muertos en pie, troncos caídos (reconocidos por su madera color pardo rojiza) y ramas desprendidas.

La ubicación de las plantas fue establecida en un mapa a escala de cada una de las parcelas. Luego, se utilizó la fórmula de Clark y Evans de la distancia al individuo más cercano (Krebs 1989) para caracterizar el patrón de distribución.

Para determinar la influencia de los rametes en la estructura de la población, se registraron como rametes potenciales (RP) a todos los brotes aéreos de una rama o tronco caído, que les correspondiera un anclaje (o raíz que penetraba en el suelo) o también a una rama de un individuo en pie que tuviera brotes radiculares con anclajes. Al entrar en contacto con el suelo, estas raíces permiten a esas ramificaciones vegetativas continuar su desarrollo independiente de la planta madre (Botina 1990). Si el brote aéreo no poseía ningún anclaje radical, no se registró como RP. A los RP se les enumeró separadamente incluyendo a todos (de rama o tronco caído). A éstos se les midió la altura, DAP o DAB, según correspondiera, y número de anclajes (NA). Es importante señalar que fue más fácil identificar a los individuos propagados a partir de rametes, únicamente en la categoría donde incluyó plántulas y brinzales, debido a que el tronco o rama que los produjo todavía no se ha descompuesto totalmente. No siempre es posible encontrar en el bosque las evidencias claras de si su origen fue por propagación vegetativa o por reproducción sexual.

Las plántulas y brinzales se observaron cualitativamente (una sola vez), tomando en cuenta su grado de herbivoría (HB, hojas comidas por 
insectos), presencia de hongos ( $\mathrm{H}$, manchas necrosadas en lámina foliar), daños mecánicos (DM, quebraduras, hojas enrolladas por acción de insectos o arañas), así como epifilia (EP). La escala utilizada fue 0 (ausencia del daño y la epifilia) hasta 4 (de 76 a $100 \%$ de presencia del daño). Estos factores se correlacionaron linealmente con la altura.

\section{RESULTADOS}

Se encontró una densidad promedio de adultos y juveniles de 1.15 (parcela 1) y 0.92 (parcela 2) ind de $W$. uxpanapensis $/ 100 \mathrm{~m}^{2}$ (Fig. 1). Las plántulas y brinzales tenían una densidad de 0.69 y 0.70 , los juveniles de 0.34 y 0.38 , y los adultos de 0.12 y $0.13 / 100 \mathrm{~m}^{2}$, en la parcela 1 y 2, respectivamente. El mayor porcentaje de plantas de $W$. uxpanapensis estuvo comprendido en la clase $\leq 1.5 \mathrm{~m}$ de altura en las dos parcelas (60.3 y $41.7 \%$ ), y el menor en las dos clases subsiguientes (Fig. 2). Se observó un incremento en la última clase ( $>10 \mathrm{~cm}$ de DAP).

En la categoría de plántulas y brinzales se registró un porcentaje muy alto (parcela 1 , $75.1 \%$ y parcela 2, $85 \%$ ) de individuos en la clase diamétrica basal de 1 a $10 \mathrm{~mm}$, mientras que la de $41 \mathrm{~mm}$ representó un $5.74 \%$, en las dos parcelas. La densidad de árboles muertos (se encontraron troncos caídos de hasta $42 \mathrm{~m}$ de longitud) fue de 11/ha en la parcela 1 y cinco en la parcela 2.

La población de $W$. uxpanapensis tenía una frecuencia total (número de subparcelas en que apareció la especie en cada parcela) de $45 \%$ (parcela 1) y 39 \% (parcela 2). En la parcela 1 la frecuencia de árboles adultos fue de 7 \% y en la parcela 2 de $11 \%$.

Para la parcela 1 se estimó una distribución aleatoria en árboles (Índice de Clark $\mathrm{y}$ Evans $(\mathrm{IC}-\mathrm{E})=1.07, \mathrm{p}<0.1, \mathrm{n}=15$ ), en individuos mayores a $5 \mathrm{~cm}$ de DAP, y en las plántulas y brinzales una distribución agrupada (IC-E $=0.028, \mathrm{p}<0.1, \mathrm{n}=18$ ). En la parcela 2, la distribución también fue aleatoria con tendencia a la distribución uniforme en árboles (IC-E $=1.34, \mathrm{p}<0.1, \mathrm{n}=18$ ), y en las plántulas

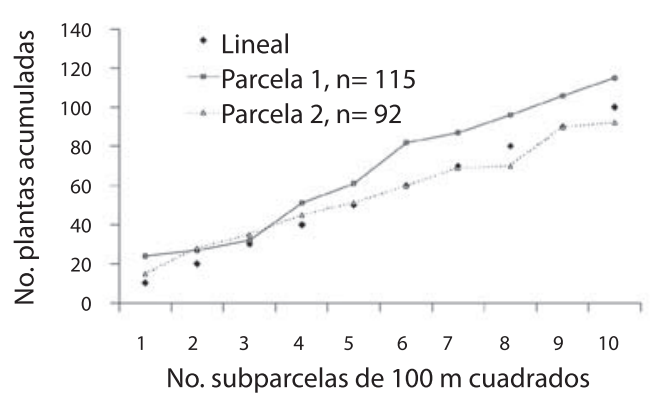

Fig. 1. Número acumulativo de individuos de W. uxpanapensis, cada100 $\mathrm{m}^{2}$, de todas las clases diametrales.

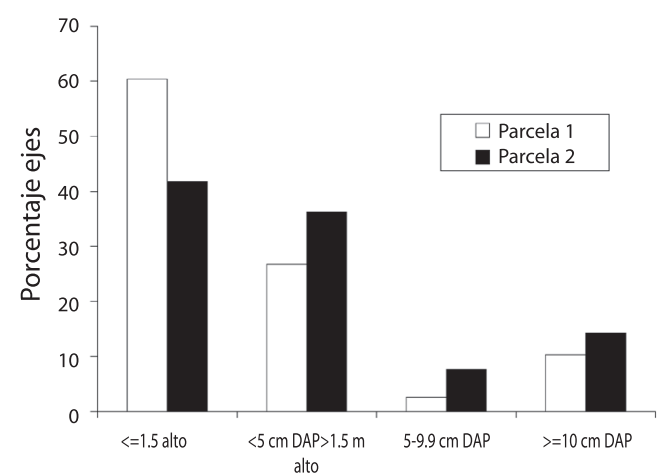

Fig. 2. Porcentaje de plantas de W. Uxpanapensis en cada categoría de altura diámetro, en las dos parcelas.

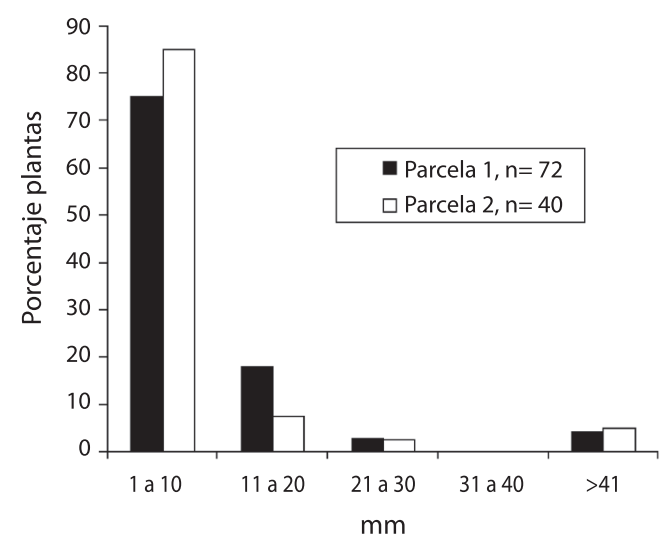

Fig. 3. Porcentaje de plántulas de W. uxpanapensis, según su altura, en cada categoría.

y brinzales, una distribución agregada (IC-E= $0.025, \mathrm{p}<1, \mathrm{n}=79$ ). La distancia promedio al vecino más cercano en los adultos fue de 14.26 m $( \pm 8.94 \mathrm{DE})$ en la parcela 1 , y de $15.33 \mathrm{~m}$ ( $\pm 5.14 \mathrm{DE})$ en la parcela 2. 
Los individuos observados de W. uxpanapensis medían desde $11 \mathrm{~cm}$ hasta alrededor de $45 \mathrm{~m}$ de altura, y se encontraron individuos desde $1 \mathrm{~mm}$ hasta $1.40 \mathrm{~m}$ de diámetro. El área basal de los siete árboles con DAP $>10 \mathrm{~cm}$, encontrados en la parcela 1 fue de $3.05 \mathrm{~m}^{2} / \mathrm{ha}$, y de $5.38 \mathrm{~m}^{2} /$ ha con 11 árboles en la parcela 2 .

W. uxpanapensis presentó gambas de $0.3 \mathrm{~m}$ de altura en individuos de $8 \mathrm{~m}$ de altura, y de hasta $4 \mathrm{~m}$ en árboles de unos $45 \mathrm{~m}$ (estos dos parámetros se correlacionaron $\mathrm{r}=0.94, \mathrm{n}=20$, $\mathrm{p}<0.01)$. Su fuste es angular, y en una sección transversal, éste es irregular. Tiene una corteza pardo-rojiza, desprendible en escamas, sin patrón de distribución definido. Se observó en un árbol recién caído de 35 m de largo, que mostraba un sistema radicular superficial, con tres raíces gruesas y el resto eran pequeñas y muy abundantes.

Las longitudes o alturas oscilaron de 2.5 a $30 \mathrm{~m}( \pm 15.81 \mathrm{DE})$. No se encontró una correlación lineal entre las categorías de diámetro y de altura, con respecto a la pendiente (parcela $1 \mathrm{r}=0.18, \mathrm{n}=115, \mathrm{p}>0.1$; parcela $2 \mathrm{r}=0.14$, $\mathrm{n}=92, \mathrm{p}>0.1$ ). En las 2 ha analizadas, $W$. uxpanapensis se ubicó tanto en pendientes suaves como abruptas (1 a 52 grados).

Se observaron troncos y ramas caídos con brotes. Estas últimas produjeron brotes aéreos al cabo de seis semanas (Fig. 4), y nueve meses después ya tenían brotes radiculares (una rama caída en abril de 1994, presentaba raíces en febrero de 1995, Fig. 5). En la parcela 2, se desprendieron tres ramas de tres árboles diferentes, cada uno con 35, 70 y 200 brotes aéreos, pero aún no poseían brotes radiculares al final de esta investigación.

También se encontró que árboles en pie mayores de $35 \mathrm{~m}$ de altura mostraban disposición a producir rebrotes en su fuste, y algunos de estos brotes parecían "saltar" hacia otros sustratos para luego producir raíces (Fig. 6, 8).

La caída de las ramas provocó la apertura de claros de hasta unos $20 \mathrm{~m}$ de radio, los cuales aparentemente facilitan que los propágulos vegetativos se establezcan y sobrevivan. Se observó que plántulas, brinzales y latizales en pie con tallo muy delgado, buscaban la luz

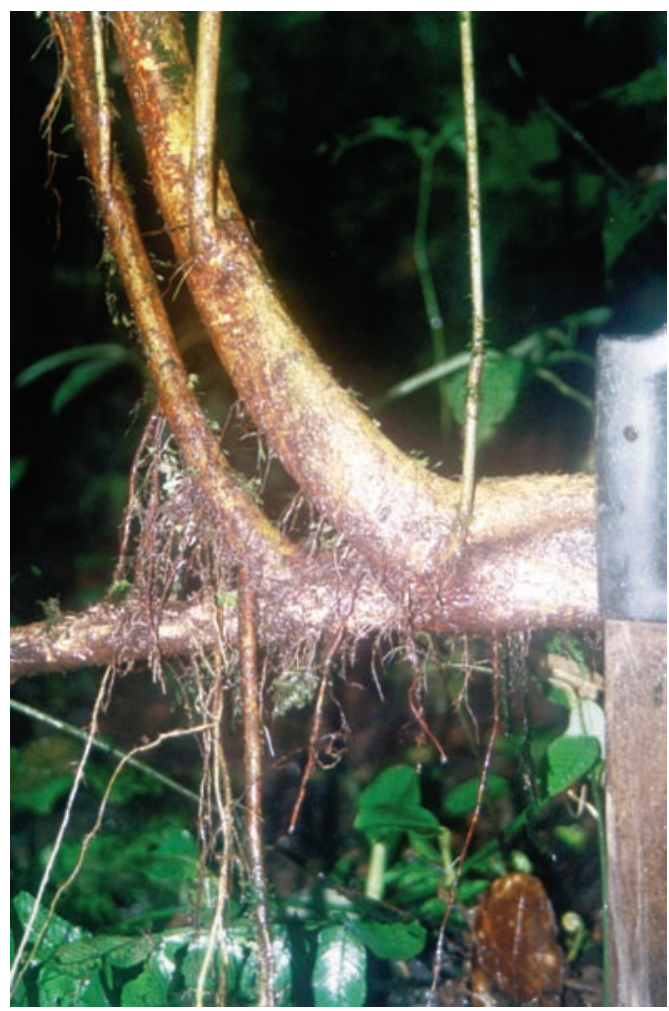

Fig. 4. Rama de W. uxpanapensis con brotes foliares, seis semanas después de desprendidas.

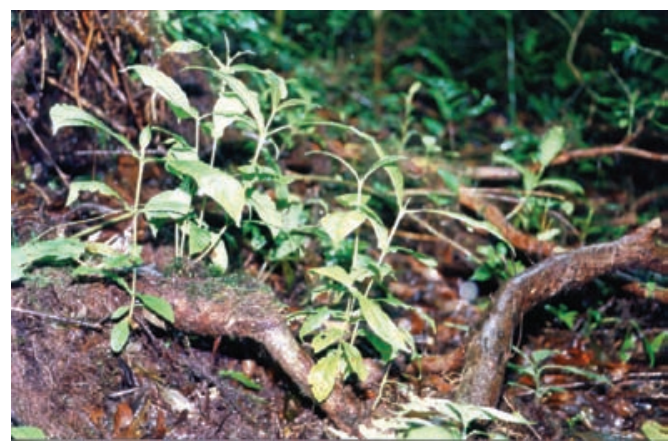

Fig. 5. Rama desprendida de W. uxpanapensis con raíces.

creciendo y fácilmente se inclinaban formando ángulos de 35 a 45 grados. Dicha inclinación estimuló, en algunos individuos, la aparición de brotes radiculares en el tallo.

Muchas de las plantas registradas en las parcelas 1 y 2 crecían sobre troncos de árboles muertos de W. uxpanapensis (Fig. 7, 8). En 


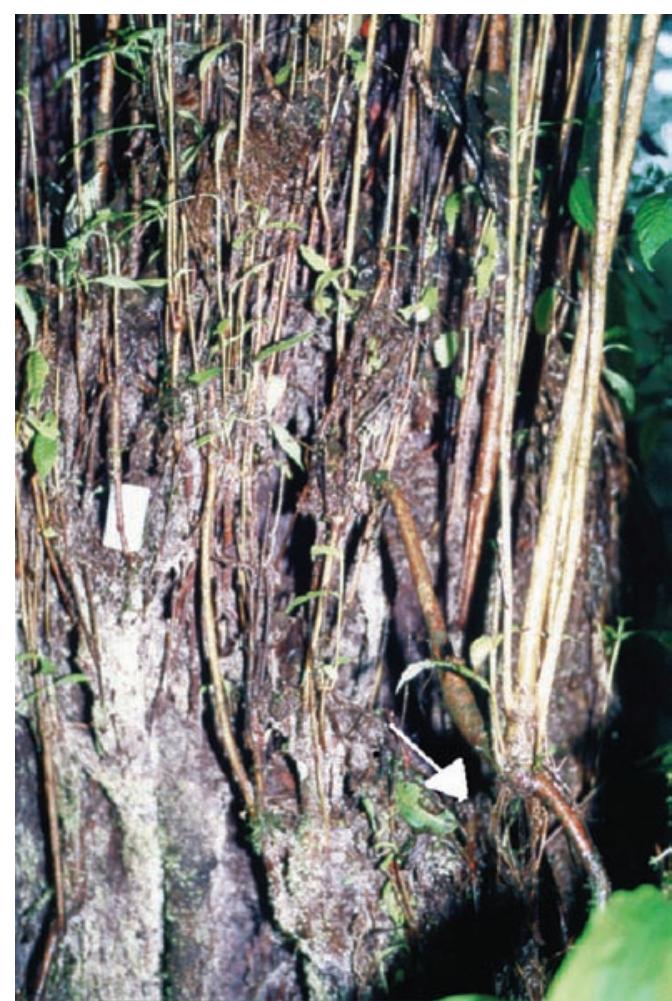

Fig. 6. Árbol en pie de W. uxpanapensis con abundantes brotes aéreos.

algunos de los 11 tocones presentes en la parcela 1 y de los cinco en la parcela 2 , se pudo observar individuos creciendo muy cercanos a ellos, y las ramas que los mantuvieron unidos al tronco, en proceso de descomposición. En la parcela 1, ocho brinzales presentaban dicha condición, y en la parcela 2, 13.

En la parcela 1 se encontró un total de 94 individuos. Si además se incluyen los 34 rametes potenciales hallados en trece unidades*, la población de plántulas aumentaría en 26.6 \% y la población total a 128 individuos. En la parcela 2 se registraron 85 individuos y 12 RP en cinco unidades, lo que corresponde a un incremento de $12.4 \%$ de las plántulas. Para esta parcela, la población podría aumentar a 97. En términos de densidad, se encontró un promedio de 0.8 y $0.31 \mathrm{RP} / 100 \mathrm{~m}^{2}$, respectivamente.

El número de RP en las ramas desprendidas, que ocurrió durante el estudio, varió entre dos y cinco, y el mayor número de ramas

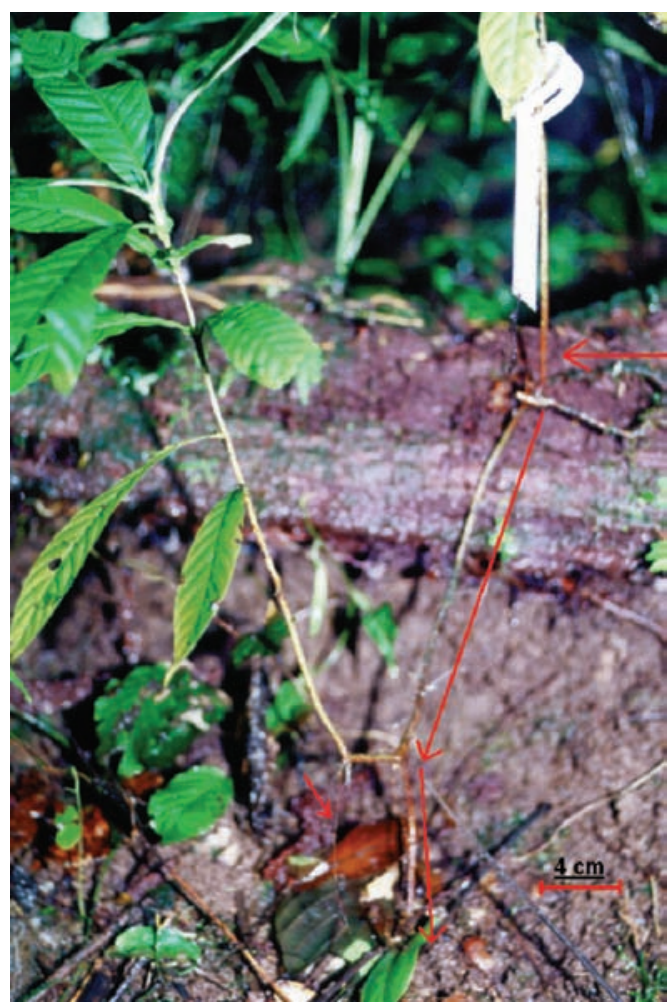

Fig. 7. Dos rametes pequeños de $W$. uxpanapensis creciendo sobre un Tocón caído de la misma especie.

desprendidas con dos rametes, se encontró en la parcela 1 (Cuadro 1).

La mayoría de las plántulas, brinzales o latizales presentaban algún daño y epifília. Aunque los valores variaron de una parcela a otra, la mayor severidad se observó para la herbivoría foliar por cortadores, y para los daños mecánicos. La epifilia fue la menos evidente,

CUADRO 1

Número de rametes potenciales (RP) por rama desprendida de $\mathrm{W}$. uxpanapensis en las dos parcelas $\operatorname{ReBAMB}, 1997-1998$

TABLE 1

Number of potential ramets per W. uxpanapensis fallen branch in both plots, ReBAMB, 1997-1998

$\begin{array}{llllll}\text { \# de RP/ rama caída } & 1 & 2 & 3 & 4 & 5 \\ \text { Parcela 1 (\%) } & 0 & 9 & 1 & 2 & 1 \\ \text { Parcela 2 (\%) } & 0 & 3 & 2 & 0 & 0\end{array}$




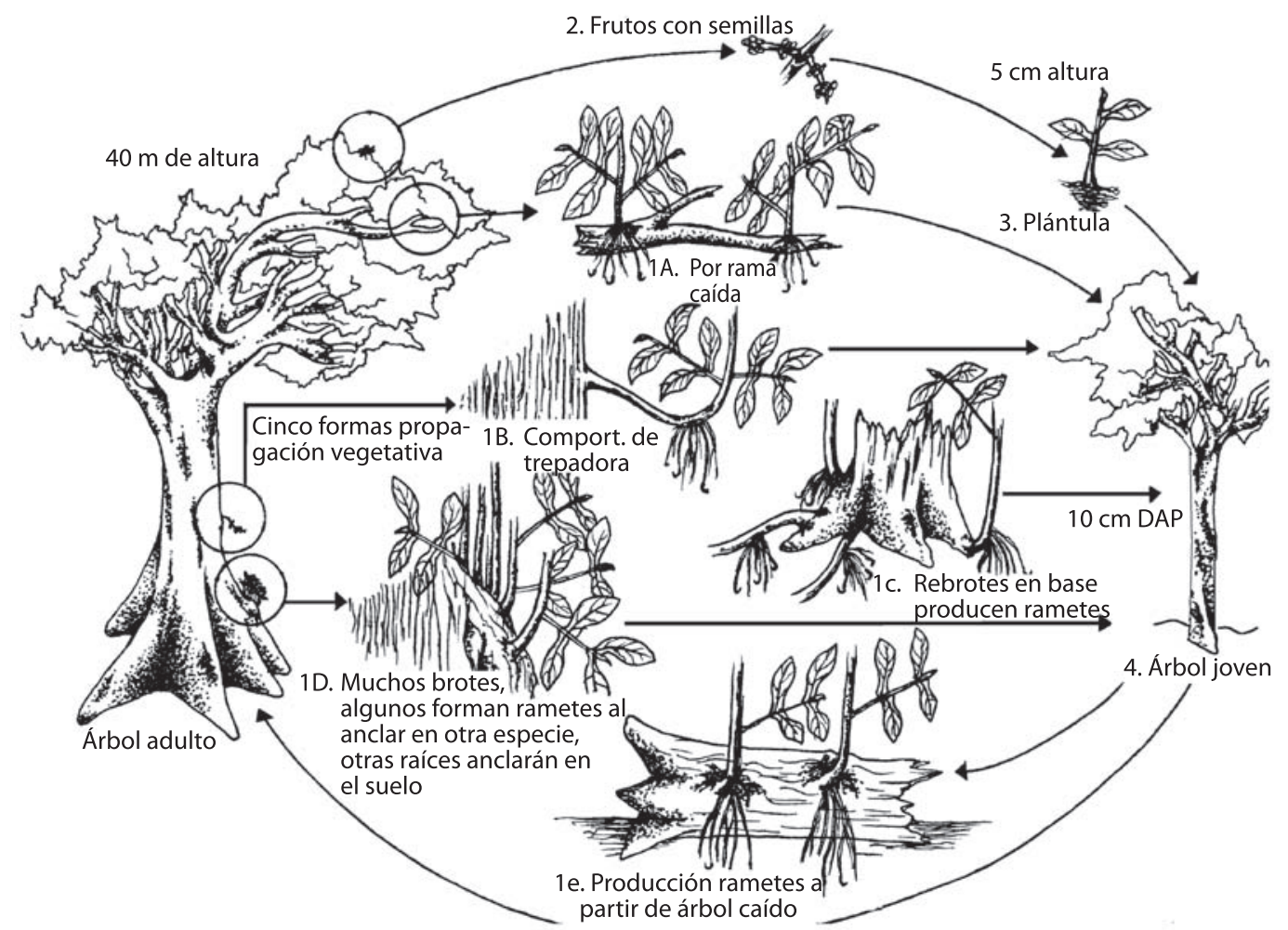

Fig. 8 Estrategia de propagación de W. uxpanapensis. Obsérvese las cinco formas de propagación vegetativa, como alternativas a la reproducción sexual.

ya que al 34.7 y $37.5 \%$ de las plantas, en las parcelas 1 y 2, no se les observó este fenómeno (Cuadro 2). Los daños no se correlacionaron linealmente con la altura de la planta, o no fueron consistentes entre las parcelas.

\section{DISCUSIÓN}

Hubbell y Foster (1987) indican que cada especie limita el crecimiento de su población más que el de las otras especies, de manera que los procesos denso-dependientes impiden a cualquier especie hacerse completamente dominante, en muchos ecosistemas. W. uxpanapensis en las dos parcelas analizadas tiene una alta dominancia en términos de su densidad, frecuencia, área basal y cobertura, considerando las categorías diamétricas. Con una densidad relativamente alta, $W$. uxpanapensis alcanzó en la ReBAMB un índice de valor de importancia del $40 \%$ con respecto a 94 especies de árboles encontrados en 1 ha (Wattenberg et al. 1996).

La densidad relativamente alta de este árbol, en este estudio, coincide con lo observado por Wattemberg et al. (1996) quienes determinaron dominante a esta especie, en los alrededores de la Estación Biológica de la ReBAMB.

La estructura de población de "J" invertida puede deberse al establecimiento de plántulas producidas por semilla, pero los rametes deben estar jugando un papel importante en este caso (se demostró que esta especie tiene una elevada capacidad para rebrotar a partir de ramas o troncos caídos, e inclusive de fustes en pie y que estos produzcan raíces, como se discutirá más adelante). La diferencia de cerca de $21 \%$ 
CUADRO 2

Porcentaje de plántulas y brinzales de W. uxpanapensis con daños y epifilia en varias categorías de severidad en la parcela $1(\mathrm{P} 1, n=70)$ y parcela $2(\mathrm{P} 2, n=38), \mathrm{ReBAMB}, 1997-1998$

TABLE 2

Percentage of $\mathrm{W}$. uxpanapensis seedlings and saplings with damage and epiphyll cover in several categories of severity in plot $1(P 1, n=70)$ and plot $2(P 2, n=38)$, ReBAMB, 1997-1998

\begin{tabular}{|c|c|c|c|c|c|c|c|c|c|c|}
\hline \multirow{2}{*}{$\begin{array}{l}\text { Severidad del daño } \\
\text { Parcelas }\end{array}$} & \multicolumn{2}{|c|}{$\begin{array}{c}0 \% \\
\text { (0 yemas dañadas) }\end{array}$} & \multicolumn{2}{|c|}{$\begin{array}{c}1-25 \% \\
(1)\end{array}$} & \multicolumn{2}{|c|}{$\begin{array}{l}26-50 \% \\
(2)\end{array}$} & \multicolumn{2}{|c|}{$\begin{array}{c}51-75 \% \\
(3)\end{array}$} & \multicolumn{2}{|c|}{$\begin{array}{l}76-100 \% \\
(4)\end{array}$} \\
\hline & $\mathrm{P} 1$ & $\mathrm{P} 2$ & $\mathrm{P} 1$ & $\mathrm{P} 2$ & $\mathrm{P} 1$ & P2 & $\mathrm{P} 1$ & $\mathrm{P} 2$ & $\mathrm{P} 1$ & $\mathrm{P} 2$ \\
\hline Herbivoría & 0 & 0 & 40.8 & 41.7 & 42.8 & 45.0 & 11.2 & 8.3 & 5.1 & 4.2 \\
\hline Hongos & 6.1 & 4.1 & 70.4 & 50.0 & 14.3 & 20.1 & 1.1 & 4.2 & 0 & 12.5 \\
\hline Daños mecánicos & 3.2 & 7.7 & 61.2 & 15.4 & 16.2 & 50.0 & 10.2 & 15.4 & 4.1 & 11.5 \\
\hline Epifilia & 34.7 & 37.5 & 48.9 & 20.8 & 13.8 & 12.6 & 3.1 & 20.1 & 0 & 0 \\
\hline
\end{tabular}

entre las parcelas 1 y 2 , en plantas menores a $1.5 \mathrm{~m}$ de altura podría deberse a que en la parcela 1 se encontraron más rametes potenciales.

La mayoría de los RP se encontraron en la categoría de plántulas con una probable alta mortalidad después. Esto podría deberse a que los RP tienen que competir por los mismos recursos de la rama madre o del suelo, o como consecuencia de mayores ataques de herbívoros o enfermedades. En este tipo de bosque, la luz resulta un factor crítico y afecta las posibilidades de que las plántulas se establezcan y sobrevivan (Römich et al. 1996). Se supone que la probabilidad de que todos los RP/unidad alcancen clases diamétricas superiores es baja, pero que al menos uno lo logre es alta debido a la gran cantidad de rebrotes producidos.

Semejante a $W$. uxpanapensis, las ramas desprendidas de E. pastoensis (Botina 1993) en Colombia, y E. auriculata (Schöers et al. 1996) en la ReBAMB cuando caen dentro de la materia orgánica que cubre el suelo, enraízan y pueden dar origen a una nueva planta. También se agrega que la regeneración natural de $E$. pastoensis puede ocurrir a partir de tocones, troncos caídos producto de la tala o derribo y de ramas caídas al quebrarse o por corte (Botina 1993). En el Alto Montclar, Colombia, y en la Sede de Occidente, Universidad de Costa Rica, se simuló artificialmente la reproducción asexual por estacas con éxito en $E$. pastoensis y W. uxpanapensis, respectivamente (Mejía 1981, Botina 1993, Brenes y Di Stéfano 1996-1997).

La capacidad de producción de brotes aéreos y radiculares en árboles en pie debe estar influenciada por cambios fenotípicos, formas de alteración, variaciones ambientales y disponibilidad de recursos (Harada et al. 1997). La plasticidad morfológica es importante en la eficiencia de obtener recursos para las plantas (Streitwolf-Engel et al. 1997). Por ello es posible que en este árbol sea una forma válida para obtener luz en un bosque con alta competencia inter-específica.

La propagación vegetativa parece ser una estrategia muy importante en este taxa, tal vez debido a que las semillas son dañadas severamente. Aunque se desconoce la efectividad de su reproducción sexual, $W$. uxpanapensis la utiliza, se localizó al menos una plántula de $7 \mathrm{~cm}$ de altura creciendo sobre un tronco caído de un helecho arborescente. Durante este estudio se recolectaron frutos directamente de un árbol, y más del $95 \%$ de las semillas estaban depredadas por el gorgojo Eurechliof sp. (Brenes y Di Stéfano, en prep.). Las semillas de esta especie, cercanas a $1 \mathrm{~mm}$ de longitud y los frutos dehiscentes, hace pensar que la dispersión de semillas sea anemócona (Brenes 1999).

W. uxpanapensis presenta alto potencial para la regeneración natural por medio de 
propagación vegetativa. Ésta podría requerir de claros producidos por la caída de ramas o árboles para el establecimiento de los rametes. Clark y Clark (1987), y Hubbell y Foster (1987) han señalado que las especies intolerantes a la sombra (umbrófilas) deben presentar poca regeneración durante mucho tiempo, porque claros grandes aparecen con mucho menos frecuencia que claros pequeños. Tal vez en esos estudios no se consideró esta estrategia de propagación.

Este comportamiento facilita la efectiva y rápida propagación vegetativa en el medio natural poco favorable, y asegura el mantenimiento de las poblaciones naturales (Zanoni 1975, Wong y Jones 1986) a través de la producción de un reservorio de RP, parecido al banco de genetes (Gavin y Peart 1999). Sin embargo, afectará la estructura genética de la población (Hamrick y Nasson 1996) haciendo que ésta sea más homogénea. Además, es un tipo de reproducción pasiva (Pulliam 1996) a corta distancia.

En las parcelas investigadas se encontró una cantidad abundante de ramas y troncos caídos. La caída de estos individuos con relativa frecuencia puede deberse a que $W$. uxpanapensis tiene la capacidad de establecerse en sitios con pendientes hasta de 44 grados, lugares que son susceptibles a la erosión y deslizamientos debido a las fuertes precipitaciones, típicas de la zona o en eventos especiales como el huracán César (ocurrido en 1996). Aparentemente cuenta con un sistema radicular superficial que le dificulta mantener un peso enorme en pie. Sprenger et al. (1996) señalan que las lluvias fuertes en pendientes escarpadas con suelos poco profundos favorecen la caída de los árboles de Plinia salticola McVauhg, en la ReBAMB.

La caída de ramas puede darse por ataques de hongos u otros patógenos, o un lento proceso de muerte (proceso interno). La combinación de esos factores, unido al hecho de que las copas de estos árboles del dosel son asimétricas, aumentan las probabilidades de desprender ramas (Van der Meer y Bongers 1996; Fig. 8), especialmente cuando se presen$\tan$ vientos fuertes.
En el caso de W. uxpanapensis, se observó una distribución agrupada en plántulas y juveniles, lo cual debe estar respondiendo a su estrategia de propagación vegetativa. Las plantas propagadas de esta manera tienden a encontrarse cerca del árbol padre, entre más alejados se encuentren, más importante es la propagación vegetativa (Harada et al. 1997).

La mayoría de las plántulas y brinzales de W. uxpanapensis se encontraron dañados por herbivoría, hongos, o/y mecánicos, aunque la severidad en éstos fue baja en su mayoría. Di Stéfano y Hurtado (1996) encontraron que un 13.5 \% de la vegetación leñosa del sotobosque de la ReBAMB, no presentó algún tipo de daño, y cuando aparecían éstos, en general, eran bajos. Tales daños podrían eventualmente causar mortalidad y provocar cambios en la dinámica de poblaciones vegetales en este bosque, por ende, afecta la abundancia y distribución de los rametes.

En un estudio realizado en la isla de Barro Colorado, con 47 especies forestales, Coley (1987) encontró bases para apoyar la teoría de que las diferencias interespecíficas, en la defensa de las plantas, puede ser explicada por el desequilibrio en la disponibilidad del carbono y nitrógeno. Datos obtenidos, por este autor con plantas de diferentes categorías de altura, muestran que las más pequeñas (posiblemente más jóvenes) se defienden mejor. Los factores bióticos son tan importantes como los abióticos en el establecimiento y distribución de la vegetación (Howe y Smallwood 1982, Clark y Clark 1985, Hubbel y Foster 1987, Sprenger et al. 1996).

La alta diversidad de especies de plantas, característica de comunidades tropicales, ha sido atribuida a patrones espaciales de la mortalidad de semillas y plántulas, como resultado de herbivoría (Janzen 1970, Connell 1971). Estudios en rametes de Piper arieianum C.DC. en La Selva, Costa Rica, realizados por Marquis (1987), afirman que la fauna herbívora daña más a ciertas plantas individuales debido a sus características genéticas.

Los daños mecánicos tuvieron mayor incidencia en las escalas desde el 1 hasta el $50 \%$ y apenas un porcentaje del 3.2 (parcela 1) y 7.7 
(parcela 2) no los presentaron. Esto es similar a lo encontrado por Di Stéfano y Hurtado (1996) en otro estudio, donde menos del $7 \%$ de las plantas del sotobosque presentaron quebraduras provocadas entre otros aspectos por el viento y la caída de material de estratos superiores.

Muchas especies han desarrollado mecanismos evolutivos (fisiológicos, demográficos y de comportamiento), o han diseñado estrategias para aprovechar los recursos y oportunidades de reproducción para provocar variaciones de distribución en el espacio y en el tiempo (Rhodes y Odum 1996).

\section{AGRADECIMIENTOS}

Agradecemos a la Vicerrectoría de Investigación, a través del Programa de Investigaciones del Bosque Premontano, y de manera especial, a Allan Artavia y a Ronald Sánchez, por su ayuda en el trabajo de campo.

\section{RESUMEN}

Se estudió los rametes, la distribución y estructura de la población de Warszewiczia uxpanapensis, en 2 ha de bosque pluvial premontano. Este árbol alcanza hasta 45 $\mathrm{m}$ de altura, DAPs hasta de $1.40 \mathrm{~m}$ y gambas de $8 \mathrm{~m}$ de altura. Su frecuencia fue de $49 \%$ y su cobertura promedio de $6.5 \%$. La distribución de los adultos fue aleatoria (distancia promedio al vecino más cercano en la parcela 1 de $14.26 \mathrm{~m}, \mathrm{IC}-\mathrm{E}=1.07, \mathrm{n}=15$ en la parcela 2 de $15.33 \mathrm{~m}$, $\mathrm{n}=18, \mathrm{IC}-\mathrm{E}=1.34)$. Sus plántulas representaron el $60.3 \%$ de la población estudiada (parcela $1, n=94$; parcela $2, n=$ 85). Se descubrió que esta especie tiene una alta capacidad de propagarse vegetativamente por rametes, los cuales influyen en la estructura de población y distribución de la especie (con una densidad de rametes parcela 1, 0.8 y parcela 2, 0.3 rametes $/ 100 \mathrm{~m}^{2}$ ). Su propagación vegetativa puede ser a partir de troncos caídos o ramas desprendidas (las cuales abren claros en el bosque), rebrotes de árboles, o ramas todavía adheridas a los árboles que son capaces de producir raíces adventicias aéreas.

Palabras clave: clon, daños, estructura de población, rametes, Rubiaceae, propagación vegetativa.

\section{REFERENCIAS}

Alpert, A. 1996. Nutrient sharing in natural clonal fragments of Fragaria chiloensis. J. Ecol. 84: 395-406.

Barnes, B.V. 1969. Natural variation and delineation of clones of Populus tremuloides and P. grandidentata in northern lower Michigan. Silvae. Genet. 18: 130142.

Begon, M. \& M. Mortimer. 1992. Population ecology, a unified study of animals and plants. Blackwell, Cambridge, Massachussetts, EEUU. 220 p.

Bolaños, R.A. \& C.V. Watson. 1993. Mapa ecológico de Costa Rica, según el sistema de clasificación de zonas de vida del mundo de L.R. Holdridge. Centro Científico Tropical, San José, Costa Rica.

Botina, J.R. 1990. El barniz o mopa-mopa, Elaeagia pastoensis Mora (Rubiaceae): estado actual de su conocimiento en Colombia. Corporación Autónoma Regional del Putumayo, Mocoa, Colombia. 86 p.

Botina, J.R. 1993. Avances sobre el conocimiento del barniz Elaeagia pastoensis Mora (Rubiaceae), en el Departamento Putumayo; período 1990-1992. Corporación Autónoma Regional del Putumayo, Colombia. 45 p.

Brenes, L. 1999. Autoecología de Elaeagia uxpanapensis D. Lorence (Rubiacaeae) en la Reserva Biológica Alberto Manuel Brenes, San Ramón, Costa Rica. Tesis de Maestría, Universidad de Costa Rica, San José, Costa Rica. 116 p.

Brenes, L. \& J.F. Di Stéfano. 1996-1997. Potencial de propagación por estacas de Elaeagia uxpanapensis. Rev. Biol. Trop. 44/45: 605-608.

Clark, D.A. \& D. Clark. 1985. Spacing dynamics of a tropical rain forest tree: evaluation of the Janzen-Connel model. Am. Nat. 124: 769-789.

Clark, D.A. \& D. Clark. 1987. Análisis de la regeneración de árboles del dosel en bosque muy húmedo tropical: aspectos teóricos y prácticos. Rev. Biol. Trop. 35(Supl.1): 41-54.

Coley, P. 1987. Patrones en las defensas de las plantas: ¿porqué los herbívoros prefieren ciertas especies? Rev. Biol. Trop. 35(Supl.1): 151-164.

Connell, J.H. 1971. On the role of natural enemies in preventing competitive exclusion in some marine animals and rain forest trees, p. 298-312. In P.J. 
den Boer \& G.R. Gradwell (eds.). Dynamics of populations. Centre for Agricultural Publishing and Documentation, Wageningen, Holanda.

Di Stéfano, J.F. \& F. Hurtado. 1996. Daños en plantas de un sotobosque pluvial, Costa Rica. Rev. Biol. Trop. 44: 275-277.

Gartner, B.L. 1989. Breakage and regrowth of Piper species in Rain forest understory. Biotropica 21: 303-307.

Gavin, D.G. \& D.R. Pearl. 1999. Vegetative life history of a dominant rain forest canopy tree. Biotropica 31: 88-294.

Hamrick, J.L. \& J.D. Nasson. 1996. Consequences of dispersal in plants, p.144-165. In O.E. Rhodes, R. Chesser \& M.H. Smith (eds.). Population dynamics in ecological space and time. University of Chicago, Chicago, Illinois, EEUU.

Harada, Y., S. Kawano \& Y. Iwasa. 1997. Probability of clonal identity: inferring he relative success of sexual versus clonal reproduction from spatial genetic patterns. J. Ecol. 85: 591-600.

Howe, H.F. \& J. Smallwood. 1982. Ecology of seed dispersal. Ann. Rev. Ecol. Syst. 13: 201-228.

Hubbel, S.P. \& R.B. Foster. 1987. La estructura espacial en gran escala de un bosque neotropical. Rev. Biol. Trop. 35(Supl.1): 7-22.

Janzen, D. 1970. Herbivores and the number of tree species in tropical forest. Am. Nat. 104: 501-508.

Krebs, C.J. 1989. Ecological methodology. Harper \& Row, Nueva York, EEUU. 653 p.

Marquis, R.J. 1987. Variación en la herbivoría foliar y su importancia selectiva en Piper arieianum (Piperaceae). Rev. Biol.Trop. 35(Supl.1): 133-150.

Mejía, G.M. 1981. El Piedemonte Putumayense; economía básica. Corporación Araracuara, Bogotá, Colombia. $55 \mathrm{p}$.

Pulliam, R. 1996. Sources and sinks: empirical evidence and population consequences, p 45-69. In O.E. Rhodes, R. Chesser \& M.H. Smith (eds.). Population dynamics in ecological space and time. University of Chicago, Chicago, Illinois, EEUU.

Rhodes, O.E. \& E. Odum. 1996. Spatiotemporal approaches in ecology and genetics: the road less traveled, p.
1-7. In O.E. Rhodes, R. Chesser \& M.H. Smith (eds.). Population dynamics in ecological space and time. University of Chicago, Chicago, Illinois, EEUU.

Richards, P.W. 1996. The tropical rain forest. Cambridge, Cambridge, Inglaterra. 575 p.

Römich, B., W. Breckle \& R. Ortiz Vargas. 1996. Morfología, fenología y exposición a la luz de Inga leonis y Pterocarpus hayesii. Rev. Pensamiento Actual 2: 21-31.

Sakai, A.K. \& T.L. Sharik. 1988. Clonal growth of male and female bigtooth aspen (Populus grandidentata). Ecology 69: 2031-2033.

Schöers, S., R. Ortiz \& S. Breckle. 1996. Apuntes sobre Elaeagia auriculata (Rubiaceae). Rev. Pensamiento Actual 2: 41-43.

Sprenger, A., S. Breckle \& R. Ortiz Vargas. 1996. Investigaciones ecológicas y demográficas sobre Plinia salticola (Myrtaceae) en un bosque húmedo premontano en Costa Rica. Rev. Pensamiento Actual 2: 45-52.

Streitwolf-Engel, R., T. Boller, A. Wiemken \& I.R. Sanders. 1997. Clonal growth traits of two Prunella species are determined by co-ocurring arbuscular mycorrhizal fungi from a calcareous grassland. J. Ecol. 85: 181-191.

Taylor, C.M. 2001. Rubiacearum Americanarum Magna Hama Pars IV: New taxa and Combinations in Elaeagia and Warszewiczia (Rondeletieae) from Mexico, Central America, and Colombia. Novon 11: 274-27.

Van der Meer, P.J. \& F. Bongers. 1996. Patterns of tree fall and branch fall in a tropical rain forest in French Guiana. J. Ecol. 84: 19-29.

Wattenberg, I., W. Breckle \& R. Ortiz Vargas. 1996. La diversidad de especies de árboles y la estructura de un bosque muy húmedo premontano en la Reserva Biológica Alberto Brenes. Rev. Pensamiento Actual 2: $11-19$.

Wong, C.Y. \& N. Jones. 1986. Improving tree form through vegetative propagation of Gmelina arborea. Commonw. For. Rev. 65: 321-325.

Zanoni M., C.A. 1975. Propagación vegetativa por estacas de ocho especies forestales. Tesis de Maestría, CATIE, Turrialba, Costa Rica. 100 p. 